\title{
Multidisciplinary Optimization Design of the Submarine based on Col- laborative Optimization Algorithm
}

\author{
Ge Hong*
}

School of Business Administration, Nanchang Institute of Technology, Nanchang city, Jiangxi province, 330099, China

\begin{abstract}
MDO (Multidisciplinary Design Optimization, MDO) is the most active field of current research on complex system engineering design. To solve the low resistance, low noise and high mobility multidisciplinary design optimization problems of submarine shape, based on multidisciplinary collaborative optimization framework, divided the optimization design of submarine shape into three disciplines multidisciplinary optimization design problem of a system-level and containing hydrodynamic discipline, flow noise discipline, maneuverability discipline. For traditional multidisciplinary collaborative optimization algorithm solving actual complex MDO problem facing the defects of convergence difficulties and calculating time-consuming, presented a realistic improved new collaborative optimization (NCO) algorithm and applied it to the multidisciplinary optimization of the submarine shape. Meanwhile, the use of response surface meta-model solved the coupling problem between discipline-level and system-level. The results show that, NCO can guarantee the stability and reliability of the submarine shape low resistance, low noise and high mobility multidisciplinary optimization design convergence results, the build of response surface meta-model between discipline-level and system-level can effectively improve the convergence rate.
\end{abstract}

Keywords: Low noise, low resistance, mobility, multidisciplinary collaborative optimization, response surface meta-model.

\section{INTRODUCTION}

The exterior design of the submarine is directly related to the resistance, self-noise of submarine and the motion stability, mobility and many other performance, and the resistance, self-noise and mobility are three very important performance indicators of the submarine, to reducing the resistance can reduce energy consumption and increase the sail and speed of the submarine; reducing the self-noise can improve the effect distance and accuracy of the sound selfguide system; improving the stability and mobility of the submarine can improve the track ability and hit probability, thus improving the operational effectiveness of the submarine. Therefore, the research on the multidisciplinary synthesized optimization design of low resistance, low noise and high mobility of the submarine appearance has very important actual significance.

However, owning to ignore the relevance of the three disciplines, the traditional serial approaches generally can only obtain the local optimal solution. Eighties of last century, the multidisciplinary design optimization (MDO) rised in the aerospace field. Scientists such as Sobieski and Kroo had done some pioneering work in this area [1-3]. MDO is a method for designing complex products and subsystems by fully exploration and using the collaborative interaction mechanism between engineering systems. With the development of MDO, a lot of optimization frames such as multidisciplinary feasible method (MDF), concurrent subspace optimization (CSSO), collaborative optimization (CO), bi-Level integrated system synthesis (BLISS), et al. Among them, the CO algorithm, proposed by Kroo et al in 1994 [2], has been considered as the most promising multidisciplinary optimization algorithm because of its good discipline autonomy and parallel processing capability.

In traditional collaborative optimization algorithms, the consistency equality constraint of system-level optimization is in the form of 2-norm. The Jacobian matrix of its derivation $\nabla J_{i}^{*}(z)=-2\left(x_{i}-\bar{z}\right)$ at its optimal solution is a bizarre matrix, which will lead to system-level Kuhn-Tucker conditions can not be met, seriously affect system-level convergence results and efficiency $[4,5]$. By changing the collaborative optimization system-level expression forms, presents an improved collaborative optimization algorithm meeting realistic projects in order to solve the defects of convergence difficulties for traditional collaborative optimization. The improved multidisciplinary collaborative optimization frame divided the low resistance, low noise and high mobility design issues of the shape of the submarine into three disciplines MDO problem of system-level and containing hydrodynamic discipline, flow noise discipline and handling discipline. The hydrodynamic discipline views reduction submarine resistance as the optimization objective, the flow noise discipline views reduction submarine flow noise as the optimization objective, and the handling discipline views increasing the submarine mobility as the optimization objective. Establish a system-level optimization model on this basis and using the precise calculation mode and approxi- 


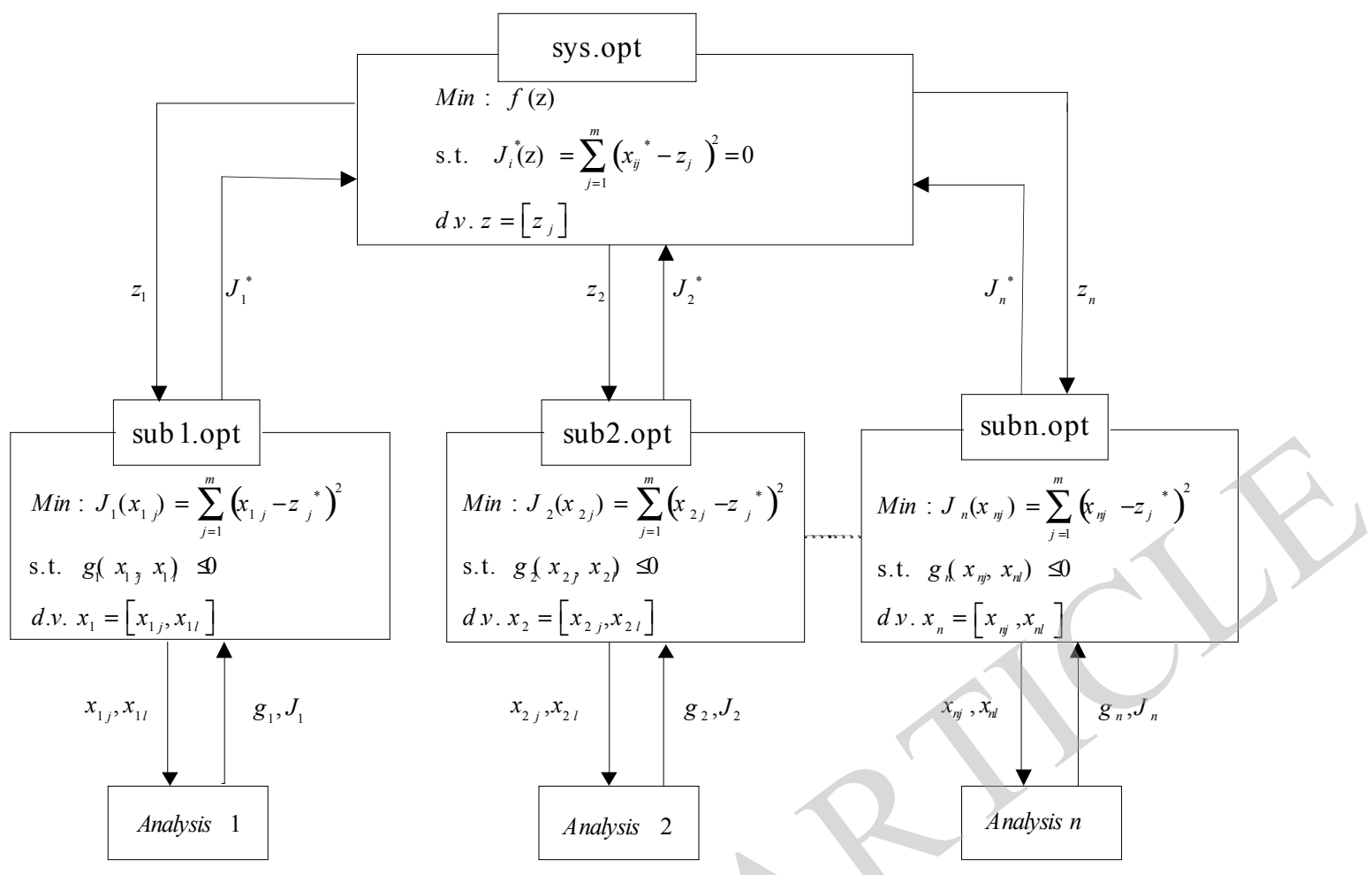

Fig. (1). Traditional CO process.

mate calculation model of the submarine flow around turbulent flow field to solve the coupling problem between the internal of discipline level, build the meta-model of each discipline level objective function in the use of response surface technology, effectively improves the convergence rate.

\section{IMPROVED COLLABORATIVE OPTIMIZATION ALGORITHM}

\subsection{Mathematical Description and Solving Process of Collaborative Optimization Algorithms}

For the inefficiency and large computational problem when the single-level multidisciplinary optimization method solves large, complex systems engineering, $\mathrm{CO}$ is proposed by Kroo, etc. as a multidisciplinary optimization strategy with two levels structure. $\mathrm{CO}$ divides the optimization problem into two levels: a system-level and parallel multidisciplinary-level. The mathematical description form of the system-level as follows:

$$
\begin{aligned}
& \operatorname{Min} f(z) \text { s.t. } J_{i}{ }^{*}(\mathrm{z})=\sum_{j=1}^{m}\left(x_{i j}{ }^{*}-z_{j}\right)^{2}=0, \quad i=1,2, \cdots n \\
& \text { d.v.z }=\left[z_{j}\right], \quad j=1,2, \cdots m
\end{aligned}
$$

where, $f(z)$ is the system-level optimization objective function; $x_{i j}{ }^{*}$ is the $j$ th shared variable optimal solution of discipline $i ; m$ is the number of shared variables of systemlevel and discipline-level; $z_{j}$ is the $j$ th shared variable; $J_{i}^{*}(\mathrm{z})$ is the consistency equation constraints provided by discipline- level $i ; n$ is the number of discipline-level of optimization problems.
The discipline-level mathematical description form of the $\mathrm{CO}$ algorithm is:

Min $J_{i}\left(x_{i}\right)=\sum_{j=1}^{m}\left(x_{i j}-z_{j}^{*}\right)^{2}, i=1,2, \cdots n$
s.t. $g\left(x_{i j}, x_{i l}\right) \leq 0, j=1,2, \cdots m$ d.v. $x_{i}=\left[x_{i j}, x_{i l}\right]$

where, $J_{i}\left(x_{i}\right)$ is the optimization objective function of discipline-level $J_{1}^{*} ; x_{i j}$ is the $j$ th shared variable of discipline $i$; $x_{i l}$ is the local variable of discipline $i ; z_{j}{ }^{*}$ is the $j$ th shared variable delivered for system-level to discipline-level $i$; $g\left(x_{i j}, x_{i l}\right)$ is the local constraints of discipline-level.

At the beginning of optimization, the system-level distributes the target value $z_{j}{ }^{*}$ of system-level variables to discipline-level, the flow chart shown in Fig. (1), each discipline-level meets their own constraint conditions, its objective function should enable the gap between the interdisciplinary coupled variables and the distributed target value is minimum, after discipline-level optimization, the optimal solution is returned to the system-level, the system-level optimizes shared variables to resolve inconsistencies between the various disciplines coupled variables in the consistency constraints. Through multiple iterations of systemlevel optimization and sub-discipline-level optimization, finally obtain the coupling relationship between disciplines; achieve a consistent system optimal design. It is this distributed parallel design ideas to ensure the freedom of various disciplines design optimization, reducing the data transfer between disciplines, attracting many scholars at home and 
abroad apply it to the design of aircraft, satellites, electric vehicles and ship [6-9].

\subsection{Improved Collaborative Optimization Algorithm}

The mathematical expression form of traditional collaborative optimization algorithm has three main issues in the real system engineering optimization design as follows [1012]:

1) The consistency equality constraint of system-level optimization is in the form of 2-norm form. The Jacobian matrix of its derivation $\nabla J_{i}^{*}(z)=-2\left(\overline{x_{i}}-\bar{z}\right)$ at its optimal solution is singular matrix, which will lead to the system level Kuhn-Tucker conditions can not be met, seriously affect system-level convergence results and efficiency.

2) For the actual complex MDO problems, the number of sub-disciplines is often more than the number of system level shared variables, so that the number of system-level constraint conditions is greater than the number of systemlevel shared variables, which greatly limits the freedom of system-level optimization.

3) System-level consistency equality constraint form, without considering the different order of magnitude difference between the design variables and design requirements for accuracy in actual engineering design, consistency constraint expression in various disciplines may cause smaller magnitude variable lose the consistency control role and too harsh equality constraints are easy to make the optimization process can not converge.

For the above analysis, proposed $\mathrm{NCO}$ algorithm, NCO framework inherited the traditional collaborative optimization distributed parallel idea, using a new system-level constraint expression form to overcome the defects of computational difficulties of traditional collaborative optimization.

1) In order to avoid the damage of system-level constraint Jacobian matrix is zero on Kuhn-Tucker conditions, using 1-norm in place of 2-norm.

2) System-level consistency constraint form changed by the expressions of greatest difference between various variables and system-level shared variables to control to enhance system-level optimization degrees of freedom, while helping to deal with the separation of the different design variables.

3) Select a different slack variables based on the actual requirements of each design variable precision.

The improved system-level optimization model is:

Min: $f_{\text {sys }}$ s.t. $\quad g_{j}=\max \left(\left|x_{11}-z_{1 j}\right|,\left|x_{21}-z_{1 j}\right|,\left|x_{n 1}-z_{1 j}\right|\right) \leq \varepsilon_{j}$

where, $j$ is the number of system-level design variables. $\varepsilon_{j}$ is the $j$ th shared variable required accuracy in actual project.

\section{MULTIDISCIPLINARY ANALYSIS MODEL OF THE SHAPE OF SUBMARINE}

The submarine used in this paper is designed to linear of the streamline rotary body expression design (shown in
Fig. 2). Submarine linear geometry parameters: total length $L=6 m$, head curve segment length $L_{M}=1 \mathrm{~m}$, cylindrical segment length $L_{H}=3.5 \mathrm{~m}$, tail total length $L_{T}=1.5 \mathrm{~m}$, maximum diameter $D_{0}=0.5334 \mathrm{~m}$, the head and tail curved segments have adopted the two-parameter elliptical line. The fin and rudder use cross symmetrical layout, the planar shape of a single fin and rudder shown in Fig. (3), wherein: $a_{f}, a_{r}$ respectively represents the span long of a fin and rudder, $b_{f}, b_{r}$ respectively represents the rudder chord of fin and rudder. The physical linear equation of head curve segment as follows:

$y=\frac{D_{0}}{2}\left[1-\left(1-\frac{X}{L_{H}}\right)^{n_{h}}\right]^{1 / \mathrm{m}_{h}}$ lows:

The physical linear equation of tail curve segment as fol-

$y=\frac{D_{0}}{2}\left[1-\left(X-L_{H}-L_{M}\right)^{n_{t}}\right]^{m_{t}}$

where, $m_{h}, n_{h}$ are double parameters of the head elliptic linear, $m_{t}, n_{t}$ are double parameters of the tail elliptic linear. Its value ranges: $2 \leq m_{h}, n_{h}, m_{h}, n_{h} \leq 5$.

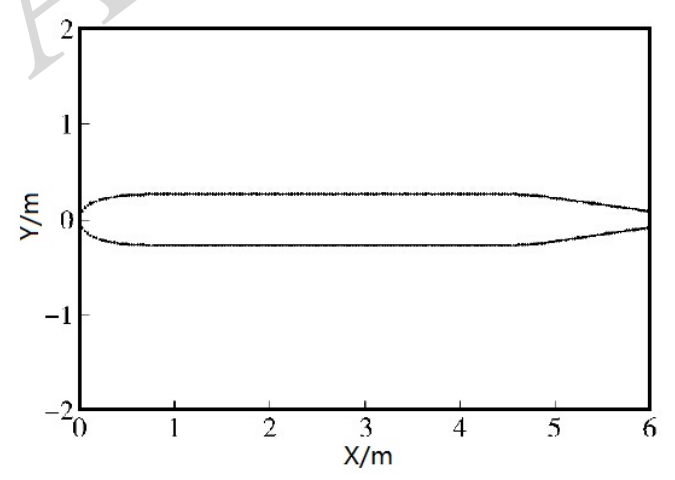

Fig. (2). Streamline rotator.

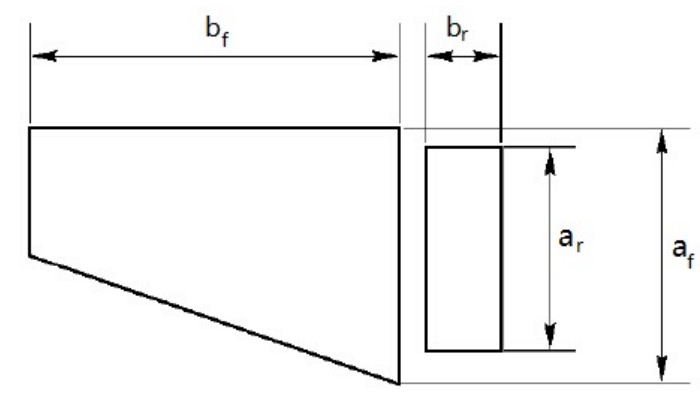

Fig. (3). Plane shape schematic of the fin and rudder.

3.1. Hydrodynamic Discipline Optimization Model

$$
\begin{aligned}
& \text { Min } F_{A}\left(\mathrm{X}_{A}, \overline{\mathrm{P}}_{\mathrm{PF}}, \overline{\mathrm{P}}_{B L}\right)=C_{x}\left(\mathrm{~m}_{h}, \mathrm{n}_{h}, \mathrm{~m}_{t}, \mathrm{n}_{t}, \overline{\mathrm{P}}_{\mathrm{PF}}, \overline{\mathrm{P}}_{B L}\right) \\
& \text { s.t. } G_{1 A}=\psi_{H}\left(\mathrm{~m}_{h}, \mathrm{n}_{h}\right) \geq 0.8 \\
& \qquad \begin{array}{l}
G_{2 A} \\
=\alpha\left(\mathrm{m}_{t}, \mathrm{n}_{t}\right) \leq 12^{0} \\
G_{3 A}=D_{E}\left(\mathrm{~m}_{t}, \mathrm{n}_{t}\right) \geq 0.1(\mathrm{~m})
\end{array}
\end{aligned}
$$


where, $\mathrm{X}_{A}$ is the design vector of hydrodynamic discipline, $F_{A}$ is the objective function of hydrodynamic discipline optimization, $G_{i A}(i=1,2,3)$ is the constraints conditions of hydrodynamic discipline, $\overline{\mathrm{P}}_{\mathrm{PF}}$ is the coupling state vector of external potential flow field, $\overline{\mathrm{P}}_{B L}$ is the coupling state vector of boundary layer. Use submarine zero wet drag coefficient $C_{x}$ as the optimization objective function, calculate as follows:

$$
C_{x}=\frac{2 \pi}{\Omega} \int_{0}^{L} C_{p} \sin (\gamma) \mathrm{YdX}+\frac{2 \pi}{\Omega} \int_{0}^{L} C_{f} \cos (\gamma) \mathrm{YdX}
$$

wherein, $C_{p}$ is the surface pressure coefficient, $C_{f}$ indicates the shear stress surface friction coefficient, $\gamma$ indicates the angle between the submarine tangent line and shaft $X, \Omega$ is the submarine wetted surface area. Among them, the around flow potential flow field uses surface element method to solve, the boundary layer uses finite difference method to solve, carry out iterative calculation between potential flow and boundary layer equations to obtain the distribution of surface pressure coefficient and surface friction shear stress coefficient.

\subsection{Flow Noise Discipline Optimization Model}

$$
\begin{aligned}
& \text { Min } F_{B}\left(\mathrm{X}_{B}, \overline{\mathrm{P}}_{\mathrm{PF}}, \overline{\mathrm{P}}_{B L}\right)=\operatorname{SPECG}\left(\mathrm{m}_{h}, \mathrm{n}_{h}, \overline{\mathrm{P}}_{\mathrm{PF}}, \overline{\mathrm{P}}_{B L}\right) \\
& \text { s.t. } G_{1 B}=\psi_{H}\left(\mathrm{~m}_{h}, \mathrm{n}_{h}\right) \geq 0.8
\end{aligned}
$$

Where, $\mathrm{X}_{B}$ is the design vector of flow noise discipline; $F_{B}$ is the objective function of flow noise disciplinary optimization; $G_{i B}(i=1)$ is the constraints conditions of flow noise discipline. Use the flow noise level radiating from submarine boundary layer transition zone to the head stagnation as optimization objective function. Based on the theory of Liepmann displacement thickness pulsating monopole sound source model [13], the power spectral density equation of boundary layer transition zone sound radiation is:

$$
\begin{aligned}
& \operatorname{SPECG}=G_{\text {monopole }}(r, \omega) \approx \frac{W \rho^{2}\left(\Delta \delta^{*}\right)^{2} u_{0} u_{c}^{2}}{8 \pi^{2} r^{2}} \times \\
& : F^{*}\left(k_{c} \Delta x, a^{*} \Delta x, \frac{u_{c} t_{i}}{\Delta x}, \frac{u_{c}}{u_{0}}\right)
\end{aligned}
$$

Among them,

$$
F^{*}\left(k_{c} \Delta x, a^{*} \Delta x, \frac{u_{c} t_{i}}{\Delta x}, \frac{u_{c}}{u_{0}}\right)=\frac{\left(k_{c} \Delta x\right)^{2}}{\left[1+\left(k_{c} \Delta x\right)^{2}\left(\frac{u_{c} t_{i}}{\Delta x}\right)^{2}\right]} \times
$$

$F\left(k_{c} \Delta x, a^{*} \Delta x, \frac{u_{c}}{u_{0}}\right)$

In the formula, $\Delta \delta^{*}$ represents the amount of change of boundary layer displacement thickness from laminar flow to turbulent flow, $\frac{u_{c} t_{i}}{\Delta x}$ represents dimensionless rise time.

\subsection{Mobility Disciplinary Optimization Model}

$$
\begin{gathered}
\operatorname{Min} F_{C}\left(\mathrm{X}_{C}, \overline{\mathrm{P}}_{\mathrm{PF}}, \overline{\mathrm{P}}_{B L}\right)=R_{z \min }\left(a_{f}, a_{r}, b_{f}, b_{r}, \overline{\mathrm{P}}_{\mathrm{PF}}, \overline{\mathrm{P}}_{B L}\right) \\
\text { s.t. } 0<G_{1 C}=G_{y}\left(a_{f}, a_{r}, b_{f}, b_{r}, \overline{\mathrm{P}}_{\mathrm{PF}}, \overline{\mathrm{P}}_{B L}\right)<1 \\
0<G_{2 C}=G_{z}\left(a_{f}, a_{r}, b_{f}, b_{r}, \overline{\mathrm{P}}_{\mathrm{PF}}, \overline{\mathrm{P}}_{B L}\right)<1
\end{gathered}
$$

Where, $\mathrm{X}_{C}$ is the design vector of mobility disciplines; $F_{C}$ is objective function of mobility disciplinary optimization; $G_{i C}(i=1,2)$ is the constraints condition of mobility disciplines. Use the minimum turning radius $R_{z \min }$ of submarine rotary movement in the horizontal plane as the optimization objective function. The formula of the minimum turning radius $R_{z \min }$ of submarine rotary movement in the horizontal plane as follows [14]:

$$
\left.\frac{L}{R_{z \min }}=\frac{\left(C_{z}^{\beta}+C_{x s}\right) m_{y}^{\beta}+C_{z}^{\delta} m_{y}^{\beta}}{\left(C_{z}^{\beta}+C_{x s}\right) m_{y}^{\omega}-\left(\mu-C_{z}^{\omega}\right) m_{y}^{\beta}} \delta_{v \max }\right)
$$

where, $\delta_{v \max }$ is the maximum rudder angle of the straight rudder; $C_{x s}$ is the maximum cross-sectional area zero lift and drag coefficient of submarine.

\subsection{System-Level Optimization Model}

$$
\begin{aligned}
\operatorname{Min} F_{S}\left(\mathrm{X}_{S}\right)=\frac{\left|\bar{F}_{A}\left(\mathrm{X}_{S}\right)-F_{A}\left(\mathrm{X}_{A}^{*}\right)\right|}{F_{A}\left(\mathrm{X}_{A}^{*}\right)}+\frac{\left|\bar{F}_{B}\left(\mathrm{X}_{S}\right)-F_{B}\left(\mathrm{X}_{B}^{*}\right)\right|}{F_{B}\left(\mathrm{X}_{B}^{*}\right)}+ \\
\text { s.t. } G_{1 S}=\psi_{H}\left(\mathrm{~m}_{h}, \mathrm{n}_{h}\right) \geq 0.8 \\
G_{2 S}=\alpha\left(\mathrm{m}_{t}, \mathrm{n}_{t}\right) \leq 12^{0} \\
G_{3 S}=D_{E}\left(\mathrm{~m}_{t}, \mathrm{n}_{t}\right) \geq 0.1(\mathrm{~m}) \\
0<G_{4 S}=G_{y}\left(a_{f}, a_{r}, b_{f}, b_{r}\right)<1 \\
0<G_{5 S}=G_{z}\left(a_{f}, a_{r}, b_{f}, b_{r}\right)<1
\end{aligned}
$$

where, $\mathrm{X}_{S}$ is the system-level design vector; $F_{S}$ is the objective function of system-level optimization; $G_{i S}(i=1,2,3,4,5)$ is system-level constraints; $\mathrm{X}_{A}^{*}$ is the optimal solution of hydrodynamic discipline optimization; $\mathrm{X}_{B}^{*}$ is the optimal solution of the flow noise discipline optimization; $\mathrm{X}_{C}^{*}$ is the optimal solution of mobility discipline optimization. $\bar{F}_{A}$ is the response surface meta-model of hydrodynamic discipline optimization objective function, $\bar{F}_{B}$ is the response surface meta-model of flow noise discipline optimization objective function, $\bar{F}_{C}$ is the response surface meta-model of mobility discipline optimization objective function.

\section{OPTMIZATION SOLUTION}

\subsection{Response Surface Meta-Model of Discipline-Level Objective Function}

1) Use the quadratic response surface method to establish the response surface meta-model $\bar{F}_{A}$ of hydrodynamic discipline objective function: 
Order: $x_{1}=m_{h}, x_{2}=n_{h}, x_{3}=m_{t}, x_{4}=n_{t}$ then:

$$
\begin{aligned}
& \bar{F}_{A}=\beta_{0}+\beta_{1} x_{1}+\beta_{2} x_{2}+\beta_{3} x_{3}+\beta_{4} x_{4}+\beta_{11} x_{1} x_{1}+ \\
& \beta_{22} x_{2} x_{2}+\beta_{33} x_{3} x_{3}+\beta_{44} x_{4} x_{4}+\beta_{12} x_{1} x_{2} \\
& +\beta_{13} x_{1} x_{3}+\beta_{14} x_{1} x_{4}+\beta_{23} x_{2} x_{3}+\beta_{24} x_{2} x_{4}+\beta_{34} x_{3} x_{4}
\end{aligned}
$$

Use Gaussian column slashing method to solve linear equations, obtain the undetermined coefficients of the response surface model are:

$\beta_{0}=-0.0073496, \beta_{1}=0.00045731$

$\beta_{2}=0.0016864, \beta_{3}=0.00143352$

$\beta_{4}=0.0015908, \beta_{11}=1.39058 \times 10^{-6}$

$\beta_{22}=-2.7649 \times 10^{-5}, \beta_{33}=-5.6105 \times 10^{-5}$

$\beta_{44}=-1.5014 \times 10^{-5}, \beta_{12}=-5.0092 \times 10^{-5}$

$\beta_{13}=-3.4226 \times 10^{-5}, \beta_{14}=-2.9776 \times 10^{-5}$

$\beta_{23}=-0.0001, \beta_{24}=-0.00023, \beta_{34}=-0.0001$

2) Use the quadratic response surface method to establish the response surface meta-model $\bar{F}_{B}$ of flow noise discipline objective function:

Order: $x_{1}=m_{h}, x_{2}=n_{h}$ then:

$\bar{F}_{B}=\beta_{0}+\beta_{1} x_{1}+\beta_{2} x_{2}+\beta_{11} x_{1} x_{1}+\beta_{22} x_{2} x_{2}+\beta_{12} x_{1} x_{2}$

Use Gaussian column slashing method to solve linear equations, obtain the undetermined coefficients of the response surface model are:

$\beta_{0}=-2263.77, \beta_{1}=605.448$

$\beta_{2}=706.75, \beta_{11}=-36.2638$

$\beta_{22}=-53.1086, \beta_{12}=-92.1491$

3) Use the response surface method to establish the response surface meta-model $\bar{F}_{C}$ of mobility discipline objective function:

Order: $x_{1}=m_{h}, x_{2}=n_{h}, x_{3}=m_{t}, x_{4}=n_{t}$ then:

$\bar{F}_{C}=\beta_{0}+\beta_{1} x_{1}+\beta_{2} x_{2}+\beta_{3} x_{3}+\beta_{4} x_{4}+\beta_{11} x_{1} x_{1}+$

$\beta_{22} x_{2} x_{2}+\beta_{33} x_{3} x_{3}+\beta_{44} x_{4} x_{4}+\beta_{12} x_{1} x_{2}$

$+\beta_{13} x_{1} x_{3}+\beta_{14} x_{1} x_{4}+\beta_{23} x_{2} x_{3}+\beta_{24} x_{2} x_{4}+\beta_{34} x_{3} x_{4}$

Use Gaussian column slashing method to solve linear equations, obtain the undetermined coefficients of the response surface model are:

$\beta_{0}=19.3052, \beta_{1}=2.3909$

$\beta_{2}=2.7131, \beta_{3}=4.9704$

$\beta_{4}=9.5031, \beta_{11}=-0.1705$

$\beta_{22}=-0.1915, \beta_{33}=-0.2541$

$\beta_{44}=-0.6939, \beta_{12}=-0.1746$

$\beta_{13}=0.0728, \beta_{14}=0.0391$

$\beta_{23}=0.0123, \beta_{24}=0.0938, \beta_{34}=-0.5417$

\subsection{Optimization Solution and Analysis}

1) System-level optimization results

The global optimization results of system-level objective function values shown in Fig. (4), we can see that from the initial solution, after 160 steps of evolution, get from the initial design to the optimal solution, optimization degree is: $98.79 \%$. The global optimal solution is:

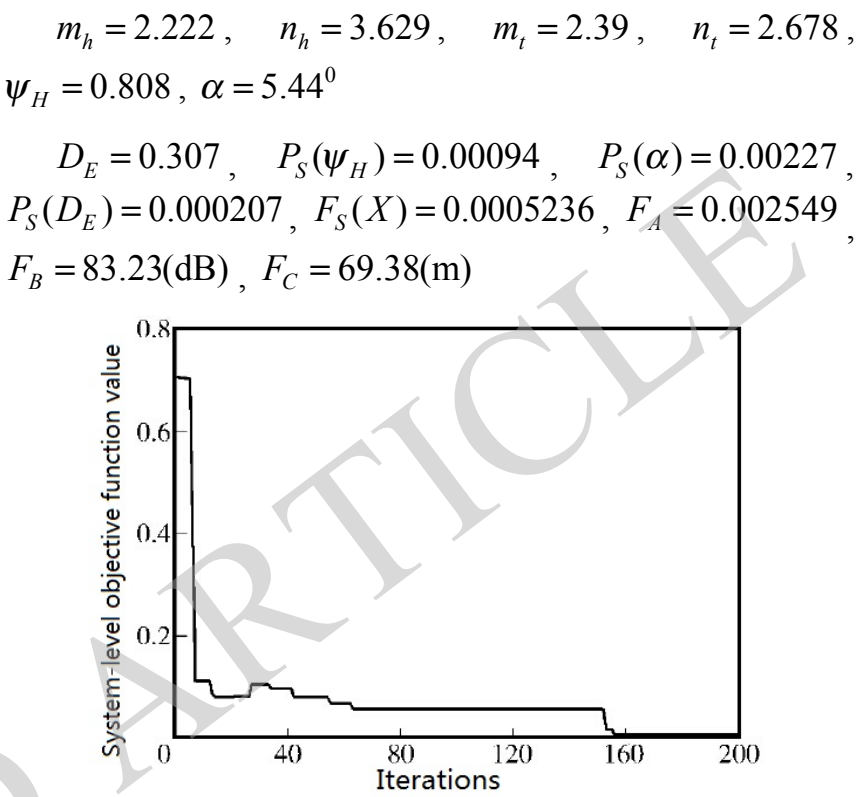

Fig. (4). Optimization results of system-level objective function values.

2) Optimization results of hydrodynamic discipline

The global optimization results of hydrodynamic discipline objective function value can be seen in Fig. (5), the optimal solution of hydrodynamic discipline-level optimization is $F_{A}=0.00254$, the optimal solution after systemlevel optimization is $F_{A}=0.00255$, the resistance increases slightly, the increased amount is $0.029 \%$. The global optimal solution is:

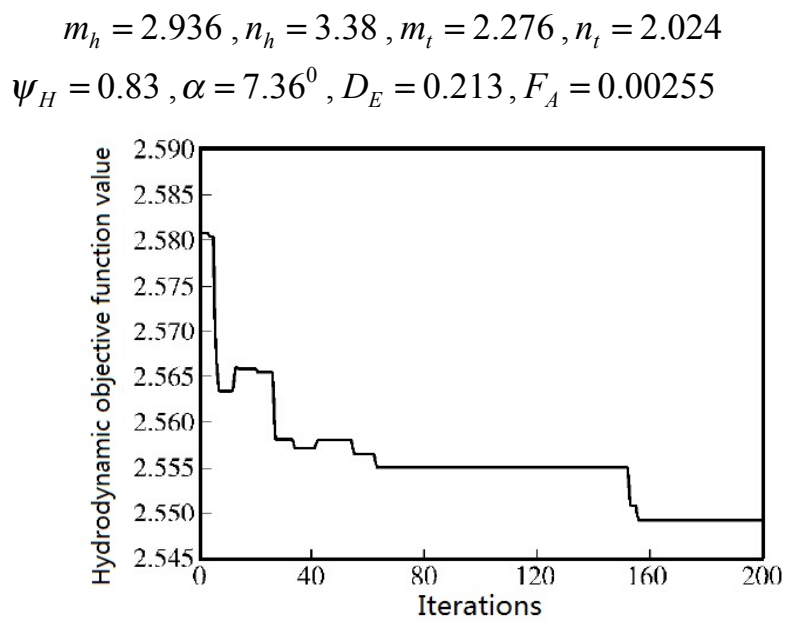

Fig. (5). Optimization results of hydrodynamic discipline objective function values. 
3) Optimization results of flow noise discipline

The global optimization results of flow noise discipline objective function value shown in in Fig. (6), the optimal solution after flow noise discipline-level optimization is $F_{B}=83.25(\mathrm{~dB})$, the optimal solution after system-level optimization is $F_{B}=83.23(\mathrm{~dB})$, flow noise is slightly reduced, the reduced amount is $0.016 \%$. The global optimal solution is:

$$
m_{h}=2.189, n_{h}=3.608, \psi_{H}=0.804, F_{B}=83.25(\mathrm{~dB})
$$

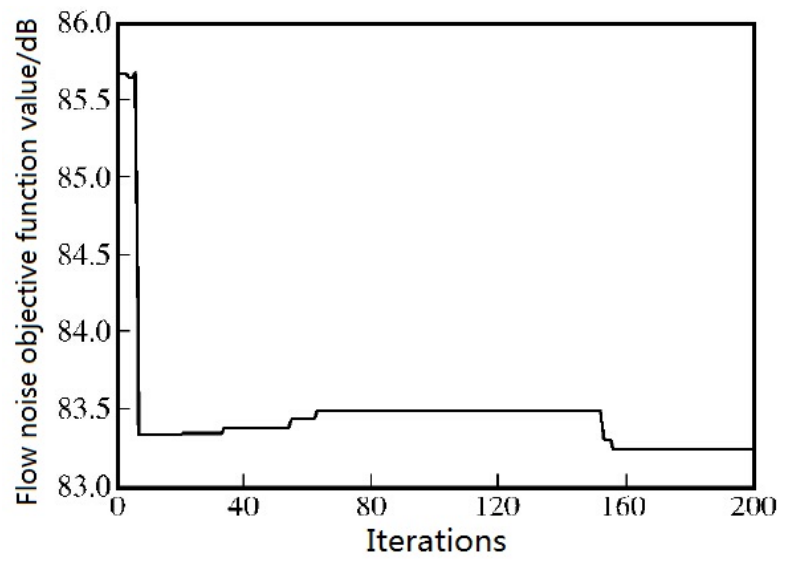

Fig. (6). Optimization results of flow noise discipline objective function values.

\section{4) Optimization results of mobility discipline}

The global optimization results of mobility discipline objective function values shown in Fig. (7). The Optimal value of mobility discipline-level optimization is $F_{C}=69.35$, the optimal solution after system-level optimization is $F_{c}=69.38$, the minimum turning radius is slightly increased, and the increased amount is $0.04 \%$. The global optimal solution is:

$$
\begin{aligned}
& a_{f}=0.2667, \alpha_{r}=0.2667, b_{f}=0.273, b_{r}=0.116, \\
& G_{z}=0.797, G_{y}=0.999, F_{C}=69.35
\end{aligned}
$$

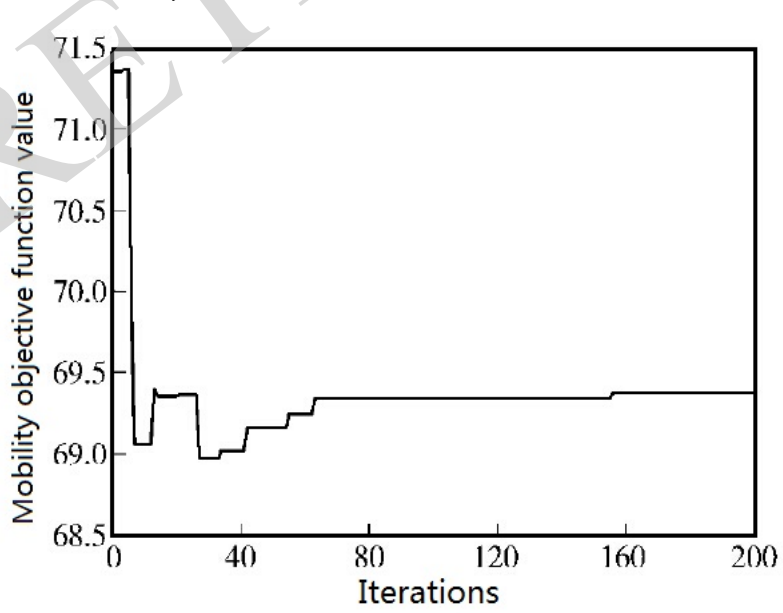

Fig. (7). Optimization results of mobility discipline objective function values.

\section{CONCLUSION}

Based on the analysis of the reason for traditional collaborative optimization solving multidisciplinary problem prone to defects, through changing the presentation of the system-level consistency constraints, an improved collaborative optimization algorithm was proposed. On the basis of inheriting the highly autonomous, parallel optimization of the design ideas of traditional collaborative optimization algorithm, NCO can effectively guarantee the existence of a viable domain solution. Based on improved collaborative algorithm, established a complete multidisciplinary comprehensive optimization design method and model for low resistance, low noise and high mobility of submarine shape, used response surface models to establish the meta-model of various discipline-level objective functions, the system-level optimization model was established entirely based on the approximate response surface model, the state variables information required in system-level optimization were obtained via response surface, this would resolve the coupling problem between system-level and discipline-level, reduced the amount of computation in system-level optimization, improved the overall optimization efficiency.

\section{CONFLICT OF INTEREST}

The author confirms that this article content has no conflict of interest.

\section{ACKNOWLEDGEMENTS}

Declared none.

\section{REFERENCES}

[1] J. Sobieszczanski-Sobieski, and R. T. Haftka, "Multidisciplinary aerospace design optimization: survey of recent developments," Structural and Multidisciplinary Optimization, vol. 14, no. 1, pp. 123, 1997.

[2] I. Kroo, S. Altus, R. Braun, P. Gage, and I. Sobieski, "Multidisciplinary optimization methods for aircraft preliminary design," $A I A A$ paper, 4325. 1994.

[3] J. Agte, O. de Weck, J. Sobieszczanski-Sobieski, P. Arendsen, A. Morris, and M. Spieck, "MDO: assessment and direction for advancement: an opinion of one international group," Structural and Multidisciplinary Optimization, vol. 40, no. 1-6, pp. 17-33, 2010.

[4] H. Li, M. X. Ma, Z. Huang, and Y. Jing, "New adaptive penalty scheme for collaborative optimization," Journal of system simulation, no. 019, pp. 6178-6182, 2009.

[5] Z. Xia, Y. Liu, W. Fan and W. Wang, "Method study of collaborative optimization with mixed variables," Journal of system simulation, vol. 23, no. 07, pp. 55-58, 2011.

[6] M. J. Daskilewicz, B. J. German, T. T. Takahashi, S. Donovan, and A. Shajanian, "Effects of disciplinary uncertainty on multiobjective optimization in aircraft conceptual design," Structural and Multidisciplinary Optimization, vol. 44, no. 6, pp. 831-846, 2011.

[7] W. P. Z. Songlin, and W. U. Guangqiang, "Multidisciplinary design optimization of vehicle body structure based on collaborative optimization and multi-objective genetic algorithm," Journal of Mechanical Engineering, vol. 2, no. 017, 2011.

[8] X. X. Yang, and W. H. Zhang, "Multidisciplinary design optimization of solid launch vehicles based on collaborative optimization," Journal of Solid Rocket Technology, vol. 6, no. 002, 2011.

[9] Q. Zhou, L. Chen, H. Xu, and W. Liu, "Application of multidisciplinary design optimization in overall design of ship," Naval Architecture and Ocean Engineering, no. 3, pp. 6-9, 2013.

[10] N. M. Alexandrov, and R. M. Lewis, "Analytical and computational aspects of collaborative optimization for multidisciplinary design," AIAA journal, vol. 40, no. 2, pp. 301-309, 2002. 
[11] W. Zhou, D. Wang, J. Sheng, and B. Guo, "Collaborative optimization of maintenance and spare ordering of continuously degrading systems," Journal of Systems Engineering and Electronics, vol. 23, no. 1, pp. 63-70, 2012.

[12] Y. X. Fang, B. Q. Zhu, and D. B. Li, "Collaborative optimization of global and local with forging multi-level and multi-objective in random environment," Computer Integrated Manufacturing Systems, vol. 18, no. 6, pp. 1182-1194, 2012.
[13] C. Bogey, C. Bailly, and D. Juvé, "Computation of flow noise using source terms in linearized Euler's equations," AIAA journal, vol. 40, no. 2, pp. 235-243, 2002

[14] F. C. Chiu, J. Guo, C. C. Huang, and J.P. Wang, "On the linear hydrodynamic forces and the maneuverability of an unmanned untethered submersible with streamlined body," Journal of Society of Naval Architects of Japan, vol. 180, pp. 241-249, 1996.

Received: June 10, 2015

Revised: July 29, 2015

Accepted: August 15, 2015

(C) Ge Hong; Licensee Bentham Open.

This is an open access article licensed under the terms of the (https://creativecommons.org/licenses/by/4.0/legalcode), which permits unrestricted, noncommercial use, distribution and reproduction in any medium, provided the work is properly cited. 